\title{
A calcineurin inhibitory protein overexpressed in Down's syndrome interacts with the product of a ubiquitously expressed transcript
}

H.C.S. Silveira,

C.A. Sommer,

A. Soares-Costa and

F. Henrique-Silva
Departamento de Genética e Evolução, Universidade Federal de São Carlos, São Carlos, SP, Brasil

\section{Correspondence}

F. Henrique-Silva

Departamento de Genética e

Evolução

Universidade Federal de São Carlos

Rodovia Washington Luiz, km 235

13565-905 São Carlos, SP

Brasil

Fax: +55-16-260-8377

E-mail: dfhs@power.ufscar.br

Research supported by FAPESP (No. 96/11018-0). H.C.S. Silveira and

C.A. Sommer are recipients of

CNPq and CAPES fellowships,

respectively.

Received May 8, 2003

Accepted February 26, 2004

\begin{abstract}
The Down's syndrome candidate region 1 (DSCR1) protein, encoded by a gene located in the human chromosome 21, interacts with calcineurin and is overexpressed in Down's syndrome patients. As an approach to clarifying a putative function for this protein, in the present study we used the yeast two-hybrid system to identify DSCR1 partners. The two-hybrid system is a method that allows the identification of protein-protein interactions through reconstitution of the activity of the yeast GAL 4 transcriptional activator. The gene DSCR1 fused to the GAL 4 binding domain (BD) was used to screen a human fetal brain cDNA library cloned in fusion with the GAL 4 activation domain (AD). Three positive clones were found and sequence analysis revealed that all the plasmids coded for the ubiquitously expressed transcript (UXT). UXT, which is encoded in human Xp11, is a 157amino acid protein present in both cytosol and nucleus of the cells. This positive interaction of DSCR1 and UXT was confirmed in vivo by mating the yeast strain AH109 (MATa) expressing AD-UXT with the strain Y187 (MAT $\alpha$ ) expressing BD-DSCR1, and in vitro by coimmunoprecipitation experiments. These results may help elucidate a new function for DSCR1 and its participation in Down's syndrome pathogenesis.
\end{abstract}

Trisomy of chromosome 21, full or partial, is a major cause of mental retardation and other phenotypic abnormalities, collectively known as Down's syndrome, a disorder affecting 1 in 700 births (1). Although most of those affected have an extra copy of chromosome 21 , some even more rare cases involving partial trisomy define a region common to all carriers of the syndrome called Down's syndrome critical region (DSCR),
Key words

- Down's syndrome

- Down's syndrome candidate region 1

- Calcineurin

- Two-hybrid system

- Ubiquitously expressed

transcript

- Protein interactions which is purportedly involved in the carriers' anomalous phenotype (2).

A gene located in this region, approximately $2 \mathrm{Mb}$ above D21S55, has been named Down's syndrome candidate region 1 (DSCR1) (3). The genomic sequence of DSCR 1 is $45 \mathrm{~kb}$, including 7 exons and 6 introns. Analyses of different cDNAs have shown that the first four exons are alternative and code for two isoforms of 171 amino 
acids and one isoform of 197 amino acids, which differ in the N-terminal, with the last 168 residues being common (4). Northern blot analyses have detected high levels of transcripts in striated skeletal and cardiac muscles, placenta, kidney, and brain $(3,4)$. In the brain, DSCR1 is predominantly expressed in neurons of the cerebral cortex, hippocampus, substantia nigra, thalamus, and medulla oblongata (5). Analysis of the DSCR1 mouse homologue expression pattern indicates that it is a developmentally regulated gene involved in neurogenesis and, if expressed aberrantly, it could affect the normal development of this organ (6). In fact, the gene is overexpressed in the brain of Down's syndrome fetuses (7).

The DSCR1 protein interacts with the serine/threonine phosphatase, calcineurin (7), involved in many cellular processes including synaptic plasticity and apoptosis of neurons (8). Moreover, DSCR1 has been assigned to a new family of conserved proteins that bind and inhibit calcineurin and also includes the human proteins ZAKI 4 (thyroid hormone-responsive gene ZAKI 4) (9) and DSCR1-like 2 (DSCR1L2) (10). These calcineurin binding proteins are characterized by a serine-proline (SP) motif(SPPXSPP) similar to that described for the nuclear factor of activated T-cell protein family, also a calcineurin substrate (11). In addition, a stretch of $\sim 80$ amino acids near the aminoterminus of these proteins shows similarity with an RNA recognition motif found in RNA and single-stranded DNA binding proteins, suggesting that DSCR1 could bind to RNA or DNA and regulate the expression of specific genes (10). Recent studies have shown that DSCR1 is a calcineurin substrate and that the SP motif may be phosphorylated. The phosphorylated state of the protein may be responsible for its subcellular location (12), possibly controlling its stability or allowing the interaction with other proteins different from calcineurin (13). Taken together, these observations motivated us to identify other proteins that interact with DSCR1, thus possibly contributing to elucidate a putative role for this protein. We have found that DSCR1 interacts with the UXT protein (ubiquitously-expressed transcript NP_705582) in a yeast two-hybrid screen and have confirmed this interaction in vitro through co-immunoprecipitation assays.

The Matchmaker two-hybrid system 3 from Clontech (Palo Alto, CA, USA) was used for screening according to the manufacturer instructions. For plasmid construction, a full-length open reading frame coding for DSCR1 isoform 4 was inserted by fusion with the GAL 4 binding domain (BD) and cMyc epitope tag using the pGBKT7 vector (Clontech).

The pGBKT7-DSCR1 vector was used to transform the yeast strain AH109 by the lithium acetate procedure. Cells were then plated onto minimal, synthetic dropout medium lacking tryptophan (SD/-Trp). The possibility of DSCR1-activated transcription and limited yeast strain growth by toxicity was tested and eliminated. The strain containing pGBKT7-DSCR1 was further transformed with a fetal brain cDNA library cloned in fusion with the GAL 4 activation domain (AD) using the pACT2 vector (Clontech), which allows growth in medium lacking leucine. Transformation yielded 404 positive colonies that were able to grow in $\mathrm{SD} /$-Leu/ -Trp/-His medium. These clones were grown at $30^{\circ} \mathrm{C}$ for 8 to 10 days and then streaked in $\mathrm{SD} /$-Leu/-Trp/-His/-Ade/X- $\alpha$-GAL medium to verify the activation of all the reporter genes (ADE2, HIS3 and LacZ).

Only 170 colonies managed to grow under these conditions. These colonies were subjected to a new phenotype test for segregation of independent clones, preventing the presence of two or more plasmids derived from the library in a single clone. Thus, positive colonies were streaked in SD/-Leu/ -Trp/-His/-Ade and X- $\alpha$-GAL. Segregation was confirmed by the observation of white and blue colonies. 
The plasmids from 52 blue colonies were isolated from the yeast and used to transform Escherichia coli DH5a cells. After purification from E. coli, the plasmids were sequenced to completion and the sequences analyzed with the BLASTX software available at NCBI (www.ncbi.nlm.nih.gov). The positive clones were confirmed by retesting the DSCR1 protein interaction through cotransformation of the interaction DSCR 1 and library DNA plasmids into the AH109 yeast strain. The putative positives were also tested against an unrelated protein (laminin C) fused to the GAL 4 DNA BD (plasmid pGBKT7Lam) and plasmid pGBKT7 alone to discard false positives. Only clones dubbed 5-2, 233 and 45-2 showed activation of reporter genes when co-transformed with BD-DSCR1 and streaked in selective medium containing $\mathrm{X}-\alpha-\mathrm{GAL}$ (Figure 1A). Those three clones carried a plasmid with a cDNA sequence that showed $100 \%$ identity to that of the UXT protein (NP_705582).

To confirm putative interactions, a yeast mating was performed as described (14) using the yeast strains AH109 (MATa) and Y187 (MAT $\alpha$ ) (Clontech). The yeast strain MATa was transformed with the pACT2UXT or pGADT7 vector (Clontech) that contains the GAL 4-AD and allows selection in SD/-Leu medium, and the MAT $\alpha$ strain was transformed with pGBKT7-DSCR1, pGBKT7-Lam or pGBKT7. Both strains were then grown in complete YPD medium and then streaked in SD/-Leu/-Trp/-His/-Ade and $\mathrm{X}-\alpha$-GAL medium. The MATa strain expressing AD-UXT was mated with MAT $\alpha$ cells expressing the BD or BD-Lam (pGBKT7 vector containing laminin $\mathrm{C}$ as a fusion protein). No colonies were visible after plating the cells onto selective medium. Similarly, no colonies were able to grow when the MATa strain expressing BDDSCR1 was mated with MATa cells transformed with the pGADT7 vector. These results indicate that the growth of diploid cells in selective medium resulted from the spe- cific interaction between DSCR1 and UXT in vivo (Figure 1B).

A co-immunoprecipitation assay was also performed to confirm the interaction in $v i$ tro. For this experiment, the pACT2-UXT vector was purified from clone 5-2 and digested with $\mathrm{BamHI}$ and $\mathrm{XhoI}$ to obtain the cDNA sequence coding for UXT. This fragment was inserted into the pGADT7 vector previously digested with $B a m \mathrm{HI}$ and $\mathrm{XhoI}$, in frame with the hemagglutinin (HA) epitope tag under the control of the $\mathrm{T} 7$ promoter (Clontech).

Expression of the $35 \mathrm{~S}$ radioactively labeled HA-UXT and c-Myc-DSCR1 proteins was done with the TNT T7-coupled reticulocyte lysate system (Promega Corp., Madison, WI, USA), according to manufacturer instructions. An aliquot of the reaction was mixed with anti-HA or anti-c-Myc antibody (Sigma, St. Louis, MO, USA) and gently
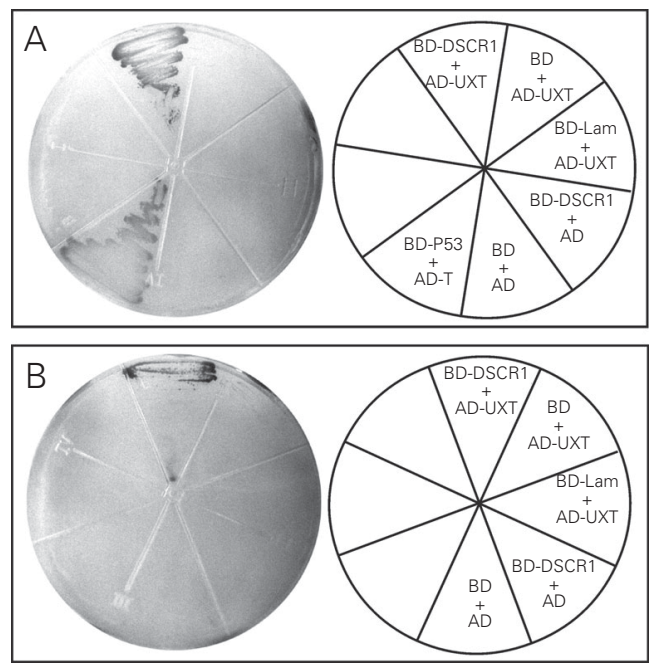

Figure 1. Analysis of the Down's syndrome candidate region 1 ubiquitously expressed transcript (DSCR1-UXT) interaction by a two-hybrid assay. A, Yeast AH109 cells were co-transformed with the pACT2-UXT plasmid expressing AD-UXT fusion and the pGBKT7-DSCR1 plasmid, which encodes BDDSCR1 fusion. Negative controls were prepared by co-transformation of AH109 cells with pACT2-UXT and pGBKT7 or pGBKT7-Lam vectors. Co-transformation with pGBKT7-DSCR1 and pGADT7 vectors was also used as a negative control. A positive control was prepared with pGBKT7-53, which expresses murine p53 fused with the GAL 4 DNA BD and pGADT7-T, expressing the SV40 large T-antigen fused to the GAL 4 AD (the proteins interact in vivo in a yeast two-hybrid screen). Transformants were streaked on plates with SD/-Leu/-Trp/-His/-Ade medium containing $\mathrm{X}-\alpha-\mathrm{GAL}$. Reconstitution of GAL 4 activity restored the ability to grow under these conditions and to detect expression of HIS3, ADE2 and LacZ reporter genes. $B$, Yeast diploids were obtained by mating AH109 transformed with the empty pGADT7 vector or pACT2-UXT with Y187 cells previously transformed with pGBKT7-DSCR1, pGBKT7 or pGBKT7-Lam vectors. Cells were streaked in SD/-Leu/-Trp/-His/-Ade and X- $\alpha$-GAL medium for diploid cell selection exhibiting reporter gene expression (AD, GAL 4 activation domain; BD, GAL 4 binding domain; AD-UXT, GAL 4 activation domain fused to UXT; BDDSCR1, GAL 4 binding domain fused to DSCR1; BD-Lam, GAL 4 binding domain fused to human laminin C; BD-P53, GAL 4 binding domain fused to murine P53; AD-T, GAL 4 activation domain fused to SV40 large T-antigen). 
rotated for $1 \mathrm{~h}$ at $4^{\circ} \mathrm{C}$. Protein A Sepharose beads (Amersham Biosciences, Piscataway, NJ, USA) were added to the tubes and washed four times with binding buffer $(50 \mathrm{mM}$ Trisbase, $150 \mathrm{mM} \mathrm{NaCl}, 1 \mathrm{mM}$ EDTA, pH 7.4, $1 \%$ Nonidet P-40, and 10\% glycerol) and once with PBS. The mixture was centrifuged and the supernatant analyzed by $15 \%$ SDSPAGE. Protein complexes were developed by autoradiography. The c-Myc-DSCR 1 protein was immunoprecipitated with the antic-Myc monoclonal antibody, and the antiHA monoclonal antibody successfully immunoprecipitated the HA-UXT fusion protein (Figure 2). As shown in Figure 2, the HA-UXT protein co-immunoprecipitated with c-Myc-DSCR1 when the anti-c-Myc antibody was present in the reaction mixture, thus confirming the results obtained by the two-hybrid assay.

DSCR1 is currently regarded as an inhibitor of calcineurin activity (15). Calcineurin binds to and dephosphorylates serine residues contained in SP repeats that are present in several proteins, including nuclear factor of activated T-cells (three SP repeats) and DSCR1 (two SP repeats) $(11,15)$. In DSCR1, the serine residues of the conserved SPPASPP motif are phosphorylated by mitogen-activated protein kinase and glycogen synthase

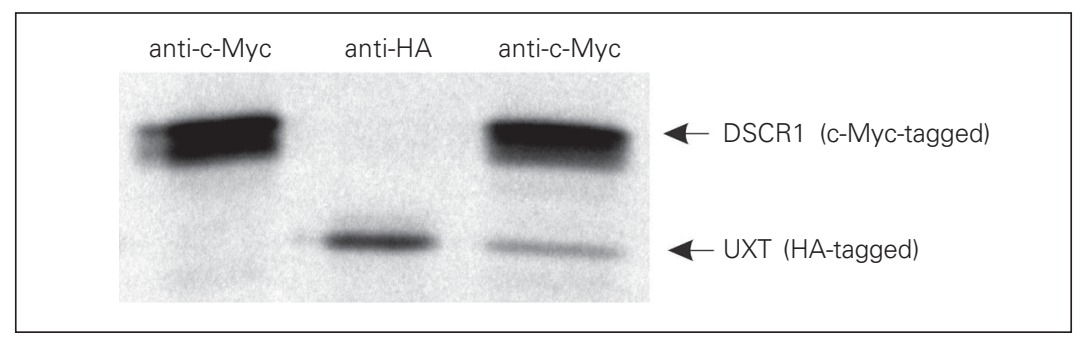

Figure 2. Gel electrophoresis showing that Down's syndrome candidate region 1 (DSCR1) co-immunoprecipitates with a ubiquitously expressed transcript (UXT) protein. cDNAs encoding c-Myc-DSCR1 and hemagglutinin (HA)-UXT were translated and simultaneously labeled with [35S]-methionine in vitro using rabbit reticulocyte lysates. They were assessed for their ability to interact and visualized by autoradiography. The c-Myc-DSCR1 and HAUXT proteins were immunoprecipitated using an anti-c-Myc antibody. kinase-3 (GSK-3). Moreover, a specific serine phosphorylated by GSK-3 has been shown to be dephosphorylated by calcineurin in vitro (13).

Current evidence suggests that the nuclear localization of DSCR 1 depends on phosphorylation. Mouse $\mathrm{C} 2 \mathrm{C} 12$ cells transfected with the DSCR1 gene fused to the green fluorescence protein show a nuclear localization of the fusion protein. It was also reported that the protein is retained in the cytoplasm when the serines of the SP repeats are mutated into alanines $(12,15)$.

Since DSCR1 is a substrate for calcineurin and protein kinases that probably regulate its cellular location, we were encouraged to identify new interactive DSCR1 partners as a first attempt to elucidate a putative function of DSCR1 in the nucleus, not previously investigated. Using the two-hybrid system, we identified an interaction between DSCR1 and the UXT protein.

UXT is a 157-amino acid protein involved in the formation of the leucine-rich pentatricopeptide repeat-motif-containing protein (LPRRPC) complex. It has been suggested that cytosolic LPRRPC protein may acquire a nuclear location which would then bridge through its primary substrates CECR2B and UXT to key chromatin-associated factors. Furthermore, the UXT protein shows a preferentially nuclear location and interacts with two other nuclear proteins, CITED2 and BUB3, that are involved in the regulation of transcription $(16,17)$. These observations, together with the results reported here, suggest that DSCR1 and UXT may interact in transcription complexes. The possibility that DSCR1 may bind to RNA or DNA and regulate the expression of specific genes has been suggested previously $(10,18)$. Whether DSCR1-UXT interaction occurs in the context of the LPRRPC complex is the subject for further investigation. 


\section{References}

1. Hassold T \& Jacobs P (1984). Trisomy in man. Annual Review of Genetics, 18: 69-97.

2. Epstein CJ, Korenberg JR, Anneren G et al. (1991). Protocols to establish genotype-phenotype correlations in Down syndrome. American Journal of Human Genetics, 49: 207-235.

3. Fuentes JJ, Pritchard MA, Planas AA, Bosch A, Ferrer I \& Estivill X (1995). A new gene from the Down syndrome critical region encodes a proline-rich protein highly expressed in fetal brain and heart. Human Molecular Genetics, 4: 1935-1944.

4. Fuentes JJ, Pritchard MA \& Estivill X (1997). Genomic organization, alternative splicing and expression patterns of the dscr1 (Down Syndrome Candidate Region 1) gene. Genomics, 44: 358-361.

5. Ermak G, Morgan TE \& Davies KJ (2001). Chronic overexpression of the calcineurin inhibitory gene DSCR1 (ADAPT78) is associated with Alzheimer's disease. Journal of Biological Chemistry, 276: 38787-38794.

6. Casas C, Martinez S, Pritchard MA et al. (2001). Dscr1, a novel endogenous inhibitor of calcineurin signaling, is expressed in the primitive ventricle of the heart and during neurogenesis. Mechanisms of Development, 101: 289-292.

7. Fuentes JJ, Genesca L, Kingsbury TM, Cunnigham KW, Perez-Riba M, Estivill X \& de la Luna S (2000). DSCR1, overexpressed in Down syndrome, is an inhibitor of calcineurin-mediated signaling pathways. Human Molecular Genetics, 9: 1681-1690.

8. Rusnak F \& Mertz P (2002). Calcineurin: form and function. Physiological Reviews, 80: 1483-1521.

9. Miyazaki T, Kanou Y, Murata Y, Ohmori S, Niwa T, Maeda K, Yamamura H \& Seo H (1996). Molecular cloning of a novel thyroid hormone-responsive gene, ZAKI-4, in human skin fibroblasts. Journal of Biological Chemistry, 271: 14567-14571.

10. Strippoli P, Lenzi L, Petrini M, Carinci P \& Zannotti M (2000). A new gene family including DSCR1 (Down Syndrome Candidate Region 1) and ZAKI-4: characterization from yeast to human and identification of DSCR1-like 2, a novel human member (DSCR1L2). Genomics, 64: 252-263.

11. Crabtree GR (1999). Generic signals and specific outcomes: signaling through $\mathrm{Ca}^{2+}$, calcineurin, and NF-AT. Cell, 96: 611-614.

12. Pfister S, Machado-Santelli G, Won Han S \& Henrique-Silva F (2002). Mutational analyses of the signals involved in the subcellular location of dscr1. BMC Cell Biology, 11: 21-24.

13. Vega RB, Yang J, Rothermel BA, Bassel-Duby $R$ \& Williams RS (2002). Multiple domains of MCIP1 contribute to inhibition of calcineurin activity. Journal of Biological Chemistry, 16: 30401-30407.

14. Finley JR \& Brent R (1994). Interaction mating reveals binary and ternary connections between Drosophila cell cycle regulators. Proceedings of the National Academy of Sciences, USA, 91: 1298012984.

15. Rothermel BA, Vega RB, Yang J, Wu H, Bassel-Duby R \& Williams RS (2000). A protein encoded within the Down syndrome critical region is enriched in striated muscles and inhibits calcineurin signaling. Journal of Biological Chemistry, 275: 8719-8725.

16. Liu L \& McKeehan WL (2002). Sequence analysis of LRPPRC and its SEC1 domain interaction partners suggests roles in cytoskeletal organization, vesicular trafficking, nucleocytosolic shuttling, and chromosome activity. Genomics, 79: 124-136.

17. Liu L, Vo A, Liu G \& Mckeehan WL (2002). Novel complex integrating mitochondria and the microtubular cytoskeleton with chromosome remodeling and tumor suppressor RASSF1 deduced by in silico homology analysis, interaction cloning in yeast, and colocalization in cultured cells. In Vitro Cellular and Developmental Biology Animal, 38: 582-594.

18. Ermak G, Harris CD \& Davies KJ (2002). The DSCR1 (Adapt78) isoform 1 protein calcipressin 1 inhibits calcineurin and protects against acute calcium-mediated stress damage, including transient oxidative stress. FASEB Journal, 16: 814-824. 